\title{
Estrategias visuales para el manejo autónomo de TIC en discapacidad intelectual severa
}

\section{Visual strategies for ICT's independent management in severe intellectual disability}

\author{
Pilar Vieiro-Iglesias*, María Luisa Gómez-Taibo*, Macarena Sánchez-Carnero*, y Teresa García-Real** \\ *Departamento de Psicología Evolutiva y de la Educación, **Departamento de Filosofía y Métodos de Investigación en Educación. \\ Universidade da Coruña
}

\begin{abstract}
Resumen
Se realizaron dos estudios con tres participantes con discapacidad intelectual severa. El objetivo era seguir un sistema horario de rutinas de ocio (estudio 1) y aprender la secuencia de pasos para manejar autónomamente TIC (estudio 2). La variable independiente fue la introducción de estrategias visuales y las variables dependientes, el número de elecciones ordenadas para usar TIC -estudio 1y el número de pasos correctamente secuenciados para realizar la actividad mediante TIC -encender, desbloquear, etc. Los resultados indican que los participantes lograron ambos objetivos. Se concluye que al ofrecer a personas con discapacidad intelectual severa información en formato accesible se favorece su autonomía.

Palabras clave: estrategias visuales, discapacidad intelectual severa, TIC, ocio.
\end{abstract}

\section{Abstract}

Two studies with three severe intellectual disabled participants were carried out. The aim was to follow a leisure routines schedule system in study 1 , and to learn the sequence of steps to independently manage TIC in study 2 . The independent variable was the introduction of visual strategies. The dependent variables were the number of ordered choices for ICT usage -study 1- and the number of correctly sequenced steps for ICT activity realization -study 2. Results showed that both aims were reached. It is concluded that when severe intellectual disabled people are offered information in an accessible format their autonomy is enhanced.

Keywords: visual strategies, severe intellectual disability, ICT, leisure.

La discapacidad no es algo estático e inmodificable, y las limitaciones funcionales que puede tener una persona se compensan por los apoyos disponibles de su entorno. Tal como afirma Schalock (2009) es necesario y determinante el apoyo a las personas con discapacidad intelectual basado en el funcionamiento individual. Una de las áreas susceptibles de apoyo es el área de la comunicación, que en las personas con discapacidad intelectual (DI) se ve limitada en cuanto a dificultades en la comprensión, adquisición y uso de los elementos morfosintácticos, en el desarrollo del lenguaje oral en todas sus dimensiones y en sus vertientes comprensiva y expresiva, etc. (Montero, 2003).

Los sistemas de comunicación aumentativa y alternativa (CAA) constituyen la elección a la hora de intervenir las limitaciones en esta área, ya que permiten a las personas con dificultades de comunicación relacionarse expresar e interactuar con su entorno, manifestando sus sentimientos, opiniones y la toma de decisiones, pudiendo participar en la sociedad en igualdad de derechos y oportunidades (Beukelman \& Mirenda, 2005). La CAA incluye diversos símbolos gráficos como objetos, fotografías, dibujos, pictogramas, palabras o letras, etc. Estos símbolos gráficos son estrategias visuales que sirven para potenciar los procesos de comunicación y para la creación de herramientas específicas para necesidades determinadas. Entre las estrategias visuales más empleadas para las personas con DI se destacan los sistemas calendario y los organizadores de tareas; estos aumentan el input lingüístico (Wood et al., 1998) al ofrecer una información lógica, estructurada y secuenciada sobre una actividad en una modalidad, la visual, que resulta accesible para ellas (Hodgon, 2006). Estos sistemas pueden utilizarse, por ejemplo, para desarrollar una rutina en una actividad determinada, para identificar la siguiente actividad a realizar, para tomar las decisiones sobre lo que puede realizarse en ese momento y para desempeñar de forma autónoma los pasos implicados en la realización de una tarea.

Cabe destacar que el aprendizaje de los sistemas de CAA debe englobarse dentro de un paradigma naturalista en donde la instrucción debe centrarse en el significado de las acciones y de lo que ocurre en contextos determinados (Kaiser, 1993). Los procedimientos naturalistas de entrenamiento del lenguaje y la comunicación se llevan a cabo dentro del entorno natural, cuando ocurren oportunidades para enseñar comunicación funcional. La enseñanza naturalista es muy eficaz proporcionando instrucciones específicas sobre formas lingüísticas básicas. Los ensayos naturalistas son breves y positivos por naturaleza. Las técnicas que se emplean para instruir desde este enfoque son el modelado, la orden modelado, la espera estructurada y la enseñanza incidental. La enseñanza de este método está enclavada en las interacciones continuas que se producen entre la persona que enseña y el aprendiz.

Por otra parte, para las personas con discapacidad intelectual el acceso a las tecnologías de la información y la comunicación (TIC) representa un importante elemento facilitador de su participación en distintos ámbitos siendo una herramienta poderosa para el aprendizaje, la participación social, el ocio y la inclusión tanto social como laboral (Pérez-Castilla, 2011) y que 
potenciarán la independencia en su vida diaria y su autonomía personal. Sin embargo, se observan obstáculos en la participación de las personas con DI ya que existen dificultades específicas para comprender y entender el medio digital, el nivel de complejidad de los contenidos y los procesos cognitivos para ofrecerle los apoyos necesarios y la creación de modelos instruccionales y metodológicos, que les permitan acceder al mundo de las tecnologías, teniendo en cuenta sobre todo los apoyos en la formación, la comunicación y las adaptaciones mediante productos de apoyo

Dada la importancia otorgada a las nuevas tecnología de la información y la comunicación (TIC) para el ocio y tiempo libre y ante la constatación de: a) la no existencia de información sobre las rutinas planificadas que impide la anticipación de los usuarios en el día a día; b) la falta de oportunidades para elegir en qué actividades de ocio participar; c) el uso y manejo de las actividades de ocio mediante TIC se produce siempre con supervisión/apoyo de un profesional, se ha planteado la realización de dos estudios con el objetivo general de mejorar la autonomía personal en cuanto a la gestión del ocio y el tiempo libre de tres personas con DI severa asistentes a un centro de día ocupacional, a través de la introducción de estrategias visuales enseñadas mediante una jerarquía de claves de instrucción decrecientes. Los objetivos específicos son: 1) Introducir estrategias visuales mediante la creación de un sistema horario para la comprensión y aprendizaje de las rutinas de ocio y tiempo libre diarias (estudio 1); 2) Introducir estrategias visuales mediante la creación de organizadores de tareas con la secuencias de pasos para el acceso a y la realización independientes de las actividades de ocio (estudio 2).

La hipótesis de partida es que el apoyo de las estrategias visuales -sistema horario y organizador de tareas- enseñadas mediante la aplicación de una jerarquía decreciente de claves instruccionales facilitará en personas con discapacidad intelectual severa el aprendizaje y comprensión de las rutinas de ocio y tiempo libre -estudio 1-, y el seguimiento de las secuencias de pasos para el uso de las TIC y la realización de actividades de forma autónoma.

\section{Método}

\section{Participantes}

Los participantes fueron dos hombres y una mujer con discapacidad intelectual severa de 35, 21 y 23 años, respectivamente. Los tres carecen de lenguaje oral y no cuentan con un sistema de comunicación simbólico; para comunicar se apoyan en el uso de señalizaciones y gestos. Asisten diariamente a la unidad de pequeño manipulado del centro de día para personas con discapacidad intelectual de la Asociación Pro Personas con Discapacidad Intelectual de Galicia (ASPRONAGA)

\section{Materiales}

3 sistemas horarios personalizados con la fotografía de cada participante y con las diferentes actividades de las sesiones de ocio y tiempo libre (pintar/puzle, tablet, pizarra digital, pantalla táctil) representadas mediante fotografías e imágenes. La Figura 1 ilustra el sistema horario del primer participante.

\section{HORARIO}

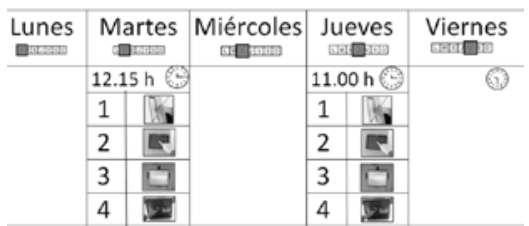

Figura 1. Sistema horario del primer participante

Cuatro organizadores de tarea, uno para cada una de las actividades de ocio y tiempo libre, con las fotografías de cada uno de los pasos implicados en la realización de la tarea.

Como tecnologías de la información y la comunicación se emplearon un tablet, una pizarra digital y una pantalla táctil

\section{Diseño y variables}

El diseño utilizado en los dos estudios fue un diseño de línea base múltiple entre sujetos (Barlow, 1988). La variable independiente para ambos estudios es la introducción de estrategias visuales (sistema horario y organizador de tareas) mediante la aplicación de una jerarquía decreciente de claves de instrucción (modelado, pointing, espera estructurada, claves naturales). La variable dependiente en el estudio 1 es el número de elecciones correctas -ordenadas- para usar la tecnología de apoyo, es decir, elección del dispositivo TIC que se va a utilizar a continuación. La variable dependiente del estudio 2 es el número pasos secuenciados correctamente para realizar la actividad mediante TIC -encender, desbloquear, etc.

\section{Procedimiento}

La investigación comenzó una vez obtenidos los consentimientos informados. Las sesiones tanto de línea base como de intervención se realizaron por las mañanas, con una frecuencia semanal de dos veces con cada participante y una duración aproximada de 30-40 minutos. La sala donde se llevaron a cabo los estudios era amplia, con una luminosidad natural y graduada para evitar el reflejo de la luz en la pantalla de los aparatos electrónicos, no existían ruidos ni distracciones. Las sesiones fueron individuales; investigadora y participante sentados a la mesa mantuvieron antes de cada sesión una pequeña conversación sobre su día con el objetivo de crear y mantener el vínculo terapeuta-usuario.

El procedimiento fue idéntico para ambos estudios. Se comenzó con el registro de la línea base a lo largo de cuatro sesiones de 30 minutos separadas entre sí veinticuatro horas.

La intervención comenzó una vez estabilizadas las observaciones en la línea base. Al comienzo de todas las sesiones se colocaba el material (sistemas horarios-estudio 1; organizador de tareas-estudio 2) encima de la mesa. En el estudio 1, todas las sesiones comenzaban con la misma pregunta "¿Qué vamos a hacer hoy?”. En el estudio 2, la frase inicial era “¿Qué es 
lo primer que tienes que hacer? La jerarquía decreciente de claves que se aplicaba si el participante no respondía era de la manera esperada: modelado - pointing - espera estructurada - claves naturales. Con el Modelado se pretende que el usuario use de manera correcta de la habilidad objetivo tras una demostración. Si tras el modelado el participante realiza la acción se retira gradualmente el apoyo para introducir pointing, consistente en señalar con el dedo la fotografía correspondiente a la siguiente actividad de ocio (estudio 1) o al siguiente paso de la actividad (estudio 2). Si con esta menor incitación el usuario señalaba correctamente la actividad o el paso siguiente, se desvanecía la ayuda de pointing dando paso a la espera estructurada consistente en introducir una demora en el tiempo para que el sujeto auto-iniciase la respuesta, hasta que finalmente las claves naturales del entorno evocasen la respuesta espontánea en el sujeto lo cual constituye un mínimo apoyo y es el objetivo. En el estudio 1, después de presentar la incitación, lo primero que tenía que hacer el usuario era despegar la primera fotografía pegada con velcro del sistema horario, por corresponder a la primera actividad de ocio a realizar, pegar dicha fotografía al lado del dispositivo de TIC y realizar la actividad. Este proceso se repite para las 3 fotografías de tecnología restantes. A cada participante se le permitía elegir las actividades de ocio que se podían realizar con cada tecnología marcada por el sistema horario mediante la selección de imágenes. Tras alcanzar los objetivos del estudio 1 y registradas las conductas objetivo en la línea base comenzaba la intervención del estudio 2. El participante tenía que seleccionar en el orden correcto cada fotografía representativa de cada paso para el manejo del dispositivo tecnológico lo que le permitía interactuar con el aparato de manera autónoma (encender, desbloquear, elegir la actividad...) y luego realizar la actividad.

\section{Resultados}

La Figura 1 permite realizar una inspección visual de los datos obtenidos en el diseño de base múltiple entre sujetos del primer estudio.

Como se puede observar, ninguno de los participantes durante la fase de línea base produjo ninguna respuesta correcta. El total de sesiones de intervención para el Participante 1 fue 11. Durante las dos primeras sesiones de intervención no produjo respuestas correctas; en la sesión 3 necesitó 4 modelos, uno por cada actividad. En la cuarta sesión, necesitó 2 modelos y 2 pointing. En la quinta sesión consiguió el $100 \%$ de las respuestas correctas. Durante la sexta sesión, necesitó tres modelos y 1 pointing. A partir de la séptima sesión y en adelante, el participante 1 obtuvo 100\% de respuestas correctas.

Por lo que respecta al Participante 2, la intervención se llevó a cabo en un total de 10 sesiones; este participante, al igual que el primero, no produjo respuestas espontáneas en las dos primeras sesiones; es a partir de la sesión 3 que produce dos respuestas espontáneas

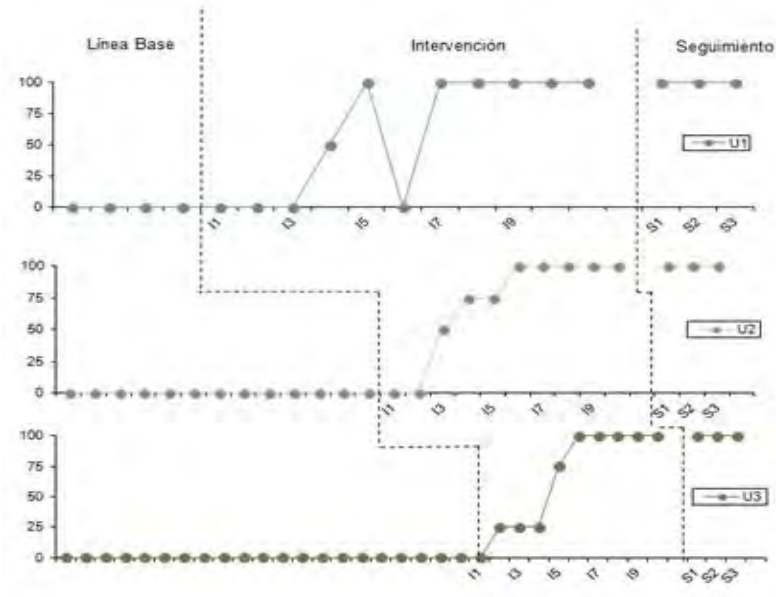

Figura 1. Elecciones de fotografías en orden correcto en el sistema horario (estudio 1)

necesitando 2 pointing para responder en las dos transiciones restantes. En las sesiones 4 y 5, precisó una incitación pointing, respondiendo ante las claves naturales en las 3 ocasiones restantes. De la sesión 6 en adelante, todas las acciones fueron espontáneas. En cuanto al Participante 3 este recibió un total de 10 sesiones de intervención. Durante la primera sesión no produjo ninguna respuesta correcta; en la segunda y tercera sesiones respondió correctamente en una ocasión aunque necesitó 3 modelados y 3 pointing respectivamente. En la sesión 4 el participante produjo una respuesta correcta espontánea y necesitó un modelado y dos pointing. En la quinta sesión solo necesitó un pointing y produjo tres respuestas correctas. A partir de la sexta a la décima sesión todas las respuestas fueron correctas y espontáneas.

En la Figura 2 se representan los resultados obtenidos por los tres participantes en el estudio 2. Como se puede observar al igual que en el estudio 1 , ninguno de los participantes durante la fase de línea base produjo ninguna respuesta correcta. El total de sesiones de intervención para el Participante 1 fue 8. Durante la primera y segunda sesiones de intervención emitió una respuesta correcta necesitando 3 modelados en la primera, y un modelado y dos pointing en la segunda. En la sesión 3, emite dos respuestas con ayuda de pointing y dos respuestas espontáneas sin necesidad de incitación. En las sesiones 4 y 5 solo precisó un pointing emitiendo en cada una tres respuestas correctas espontáneas. A partir de la sesión 6 todas las respuestas fueron correctas y espontáneas.

El Participante 2 recibió un total de 8 sesiones de intervención; pasó de necesitar 3 modelados en la primera sesión (una respuesta correcta solamente), a necesitar dos modelados y un pointing en la segunda (una sola respuesta correcta), a dos pointing en la tercera (dos respuestas correctas), y un único pointing en las sesiones cuarta y quinta (tres respuestas correctas). A partir de la 
sexta sesión, el participante 2 responde correctamente libre de incitaciones.

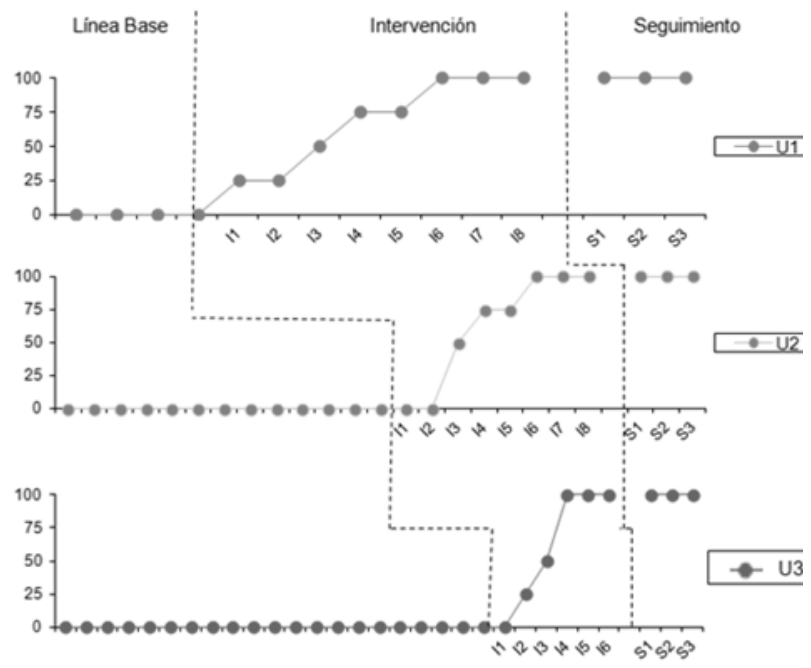

Figura 2. Elección de fotografías representando secuencias de pasos correctas (estudio 2)

Por último, la intervención para el Participante 3 se desarrolló a lo largo de 6 sesiones. En la primera sesión necesitó todos los ensayos modelados. En la sesión 2 recibió tres incitaciones pointing y produjo una respuesta correcta de manera espontánea. En la sesión 3 se incitó con pointing en dos ensayos y en los dos restantes respondió espontáneamente. A partir de la sesión 4 y en adelante realiza las acciones de manera espontánea.

También se puede observar que en las tres sesiones de seguimiento al mes de finalizada la intervención se mantuvieron los resultados de los dos estudios.

\section{Discusión}

El objetivo de este estudio es llevar a cabo una intervención con tres personas con discapacidad intelectual severa para el aprendizaje y seguimiento de un sistema horario (estudio 1) y el acceso y manejo autónomo de actividades de ocio (estudio 2) mediante un procedimiento basado en la aplicación de una jerarquía decreciente de claves de instrucción. A la vista de los resultados de ambos estudios esta intervención ha resultado satisfactoria. El conjunto de datos refleja que los participantes han recibido y se han beneficiado de los procedimientos de modelado y pointing para el aprendizaje de un sistema horario y de la secuenciación de los pasos de diversas actividades.

Por lo que respecta al Estudio 1, los tres participantes antes del inicio de la intervención no eran capaces de reconocer qué actividades tenían que realizar en su vida diaria, sin poder anticiparse a sus rutinas y limitando su autonomía y la exploración de su entorno. Sin embargo, tras la aplicación del procedimiento de este trabajo de investigación se ha podido comprobar que en 11 sesiones de intervención el Participante 1 aprendió a ordenar sus rutinas diarias con las nuevas tecnologías para el acceso a su ocio con la aplicación de un sistema calendario y unas instrucciones para su comprensión. Los Participantes 2 y 3 necesitaron 10 sesiones de intervención. No solo se ha tenido éxito en el aprendizaje sino que un mes después de la intervención los resultados se mantienen observándose los resultados en las sesiones de seguimiento.
Cabe destacar que con un mínimo de 11 sesiones se ha logrado el aprendizaje de las conductas objetivo por parte de personas que llevan recibiendo servicios de educación especial desde la infancia y que hasta el momento no habían recibido este tipo de intervención. Muchas veces las personas con DI severa no son capaces de realizar ciertas actividades porque existen limitaciones en la comprensión del funcionamiento de las mismas porque no existen oportunidades de comunicación, o bien porque existen barreas de acceso. Cuando se aumenta el input y en este sentido los sistemas horarios son el primer componente del aumento del input (Wood et al.1998), estos desajustes potenciales entre lo que se pide hacer en una tarea y la respuesta del usuario se reducen, solventándose ciertas limitaciones y permitiendo a la persona actuar de forma independiente y valerse de sí misma en la realización de las mismas.

La intervención que se ha llevado a cabo ha facilitado, por una parte, la adquisición de la comunicación simbólica, y por otra, la provisión de una visión global de la secuencia de actividades a lo largo del día, la obtención de información específica sobre lo que va a suceder a continuación en una modalidad accesible a los participantes, también una facilitación de las transiciones de una actividad a la siguiente y a la vez ha servido como componente de un plan de apoyo conductual ante la necesidad de predictibilidad todo ello en consonancia con lo que Beukelman \& Mirenda (2005) destacan.

Respecto al Estudio 2, los participantes han logrado ir de forma independiente a la mesa, coger la fotografía y ejecutar la actividad. Por lo que respecta a la ejecución de los pasos dentro de la propia actividad, los participantes en 8,8 y 6 sesiones, respectivamente, aprendieron a manejar las TIC con un sistema de apoyos de imágenes reales que especificaban los pasos a seguir para el uso de las mismas. Tal como afirma Hodgon (2006) las personas con discapacidades con frecuencia necesitan el apoyo de la información presentada en formato visual lo que les permite aprender mejor a través de lo que ellos ven. Y esto es lo que se ha logrado en este estudio.

Como conclusión, la clave del éxito ha sido la provisión de información en una modalidad, la visual, accesible a las capacidades cognitivas de los participantes junto con la aplicación de las incitaciones en orden decreciente que han permitido transferir el control hacia situaciones más naturales, todo lo cual ha permitido un acceso independiente al ocio y una mejora de la autonomía personal.

\section{Referencias}

Barlow, D., \& Hersen, M. (1988). Diseñoes experimentales de caso único. Barcelona: Martínez Roca.

Beukelman, D. R., \& Mirenda, P. (2005). Augmentative and Alternative Communication: Supporting children and adults with complex communication needs. Baltimore: Paul H. Brookes.

Hodgon, L. A. (2006). Visual strategies for improving communication. Practical supports for school and home. Michigan: QuirkRoberts Publishing 
Kaiser, A. P. (1993). Functional language. In M. E. Snell (Ed.), Instruction of students with severe disabilities. New York: Macmillan.

Montero, D. (2003). Conducta Adaptativa y Discapacidad aquí y ahora: algunas propuestas para mejorar la práctica profesional. Siglo Cero, 34(2), 68-77.

Perez-Castilla, L. (2011) Las TIC ante la diversidad: situación actual y retos del futuro. Revista de Autonomía Personal, 1(1), 46-51.

Schalock, R. L. (2009) La nueva definición de la Discapacidad Intelectual, apoyos individuales y resultados personales. Siglo Cero, 40 (229), 22-39.
Wood, L., Lasker, J., Siegel, E., Beukelman, D., Ball, L. (1998) Input Framework for Augmentative and Alternative Communication. Augmentative and Alternative Communication, 14, 261-266.

\section{Agradecimientos}

Agradecemos sinceramente al personal del Centro de Día Lamastelle (ASPRONAGA) su voluntad de colaboración para el desarrollo del presente estudio. 